\title{
Cosmogenic activation of materials
}

\author{
S. Cebrián \\ Laboratorio de Física Nuclear y Astropartículas, Universidad de Zaragoza, 50009 Zaragoza, Spain \\ Laboratorio Subterráneo de Canfranc, 22880 Canfranc Estación, Huesca, Spain
}

\begin{abstract}
The production of radioactive isotopes in materials due to exposure to cosmic rays can become an hazard for experiments demanding ultra-low background conditions. Generation of long-lived products by cosmic nucleons at sea level has been studied for detector materials like germanium and tellurium and for other materials commonly used like copper; the main results will be summarized, considering both measurements and calculations. The isotope production cross sections and the cosmic ray spectrum are the two main ingredients when calculating this cosmogenic activation; the different alternatives for implementing them will be discussed. But cosmogenic activation can take place also deep underground due to cosmic muons, being relevant in this case the short-lived products. Studies carried out to evaluate the underground activation mainly for liquid scintillator materials will be commented too.
\end{abstract}

Keywords: Activation, cosmic rays, production cross sections

PACS: 13.85.Tp; 25.40.-h; 25.30.Mr

\section{ACTIVATION AT SEA LEVEL}

Cosmogenic activation of materials is one of the common background sources in sensitive experiments underground $[1,2]$. The activity $A$ of a particular isotope of decay constant $\lambda$ induced in a material must be evaluated taking into account the times of exposure and cooling $\left(t_{\text {exp }}, t_{\text {cool }}\right)$ and the production rate $R$ :

$$
A=R\left[1-\exp \left(-\lambda t_{\text {exp }}\right)\right] \exp \left(-\lambda t_{\text {cool }}\right)
$$

As it will be shown, there are some direct measurements of productions rates in some materials just as the saturation activity, but they are not very common. Consequently, in many cases production rates have to be estimated from two basic energy-dependent ingredients, the flux $\phi$ of cosmic rays and the cross-section $\sigma$ of isotope production:

$$
R \propto \int \sigma(E) \phi(E) d E
$$

There are several possibilities to obtain values of production cross sections:

- Experimental results can be found at EXFOR database (CSISRS in USA) [3], an extensive compilation of nuclear reaction data from thousands of experiments. Available data for a particular target, projectile, energy or reaction can be easily searched for by means of a public web form.

- Semiempirical formulae give nucleon-nucleus cross sections for different reactions (break-up, spallation, fission,...) exploiting systematic regularities and tuning parameters to best fit available experimental results. The famous Silberberg \& Tsao equations [4]-[6] can be applied for light and heavy targets $(A \geq 3)$, for a wide range of product nuclides $(\mathrm{A} \geq 6)$ and at energies above $100 \mathrm{MeV}$. Their main limitation is that they are based only on proton-induced reactions; therefore, the fact that cross sections are equal for neutrons and protons has to be assumed. These equations have been implemented in different codes, which offer very fast calculations in contrast to Monte Carlo simulations:

- COSMO [7] is a FORTRAN program with three modes of calculation: excitation curve of a nuclide for a specified target, mass yield curve for given target and energy and final activities produced for a target exposed to cosmic rays. It allows a complete treatment for targets with $\mathrm{A}<210$ and $\mathrm{Z}<83$.

- YIELDX [6] is a FORTRAN routine to calculate the production cross-section of a nuclide in a particular target at a certain energy. It includes the most recent updates of the Silberberg \& Tsao equations.

- ACTIVIA [8] is $\mathrm{C}++$ computer package to calculate target-product cross sections as well as production and decay yields from cosmic ray activation. It uses semiempirical formulae but also experimental data tables if available.

Low Radioactivity Techniques 2013 (LRT 2013)

AIP Conf. Proc. 1549, 136-141 (2013); doi: 10.1063/1.4818094

(C) 2013 AIP Publishing LLC 978-0-7354-1174-6/\$30.00 
- Monte Carlo simulation of the hadronic interaction between nucleons and nuclei can output also production cross sections. Modeling isotope production includes a wide range of reactions: the formation and decay of a long-lived compound nucleus at low energies, while in the $\mathrm{GeV}$ range the intranuclear cascade (INC) of nucleonnucleon interactions is followed by different deexcitation processes (spallation, fragmentation, fission,...). Many different models and codes implementing them have been developed in very different contexts (studies of comic rays and astrophysics, transmutation of nuclear waste or production of medical radioisotopes for instance): BERTINI, ISABEL, LAHET, GEM ,TALYS, HMS-ALICE, INUCL, LAQGSM, CEM, ABLA, CASCADE, MARS, SHIELD, ... are the names of just a few of them. Some of these models have been implemented in general-purpose codes like MCNPX, GEANT4 and FLUKA. The suitability of a code to a particular activation problem depends on energy, targets and projectiles to be considered. Some systematic and extensive comparisons of calculations and available measurements have been made based on analysis of deviation factors $F$, defined as:

$$
F=10^{\sqrt{d}}, \quad d=\frac{1}{N} \sum_{i}\left(\log \sigma_{e x p, i}-\log \sigma_{c a l, i}\right)^{2}
$$

being $N$ the number of pairs of experimental and calculated cross sections $\sigma_{\text {exp }, i}$ and $\sigma_{c a l, i}$ at the same energies. For example, in [9] preferred models for each target mass range are selected, for neutron and proton-induced reactions; in [10] it is concluded that versions of CEM03 and INCL+ABLA codes can be considered as the most accurate.

- Several libraries of production cross sections have been prepared combining calculations and experimental data, with different coverage of energies, projectiles and reactions:

- RNAL (Reference Neutron Activation Library) [11] is restricted to 255 reactions.

- MENDL-2 and MENDL-2P (Medium Energy Nuclear Data Library) [12] were prepared for neutrons up to $100 \mathrm{MeV}$ and protons up to $200 \mathrm{MeV}$, based on calculations using codes of the ALICE family.

- An evaluated library for neutrons and protons to $1.7 \mathrm{GeV}$ was presented in [13].

- HEAD-2009 (High Energy Activation Data) [9] is a complete compilation of data for neutrons and protons up to $1 \mathrm{GeV}$. The choice of models was dictated by an extensive comparison with EXFOR data.

Relevant activation processes on Earth surface are induced mainly by nucleons at MeV-GeV energy range. Since protons are absorbed by the atmosphere, neutrons dominate the contribution to the cosmic ray spectrum to be considered at sea level. Different forms of the neutron spectrum at sea level have been used in cosmogenic activation studies, like the Hess [14] and Lal \& Peters [15] spectra. In [16], a compilation of measurements was made, including the historical Hess spectrum and relevant corrections, and an analytic function valid from $10 \mathrm{MeV}$ to $10 \mathrm{GeV}$ was derived (referred hereafter as Ziegler spectrum). In [17] a set of measurements of cosmic neutrons on the ground across the USA was accomplished using Bonner sphere spectrometers; a different analytic expression fitting data for energies above $0.4 \mathrm{MeV}$ was proposed (referred as Gordon et al spectrum). Both parameterizations, applied for the conditions of New York City at sea level, are compared in figure 1; the integral flux from $10 \mathrm{MeV}$ to $10 \mathrm{GeV}$ is $5.6 \times 10^{-3} \mathrm{~cm}^{-2} \mathrm{~s}^{-1}$ for [16] and $3.6 \times 10^{-3} \mathrm{~cm}^{-2} \mathrm{~s}^{-1}$ for [17].

\section{Germanium}

Different activation studies have been performed for germanium, mainly within double beta decay experiments.

- Early estimates of production rates were made in $[18,19]$ using excitation functions calculated with a spallation reaction code and the Hess and Lal \& Peters neutron spectra. Production rates were also derived experimentally in Homestake and Canfranc laboratories from germanium detectors previously exposed (table IV in [18]). Agreement with calculation was within a factor of 2 and in some cases within $30 \%$.

- Estimates of production rates for ${ }^{60} \mathrm{Co}$ and ${ }^{68} \mathrm{Ge}$ were made using excitation functions calculated with the SHIELD code in [20]. It is worth noting than in this estimate rates were evaluated including not only the neutron but also the proton contribution; in some cases the latter amounts to up to a $\sim 10 \%$ of the total (tables 2 and 3 in [20]).

- Another estimate of production rates can be found in [21]. Excitation functions were calculated using the TALYS code and neutron spectrum was considered from the Gordon et al parametrization. Even the production of ${ }^{3} \mathrm{H}$ was quantified in this work (table 1 in [21]), thanks to improvements in the availability of reaction codes that fully 


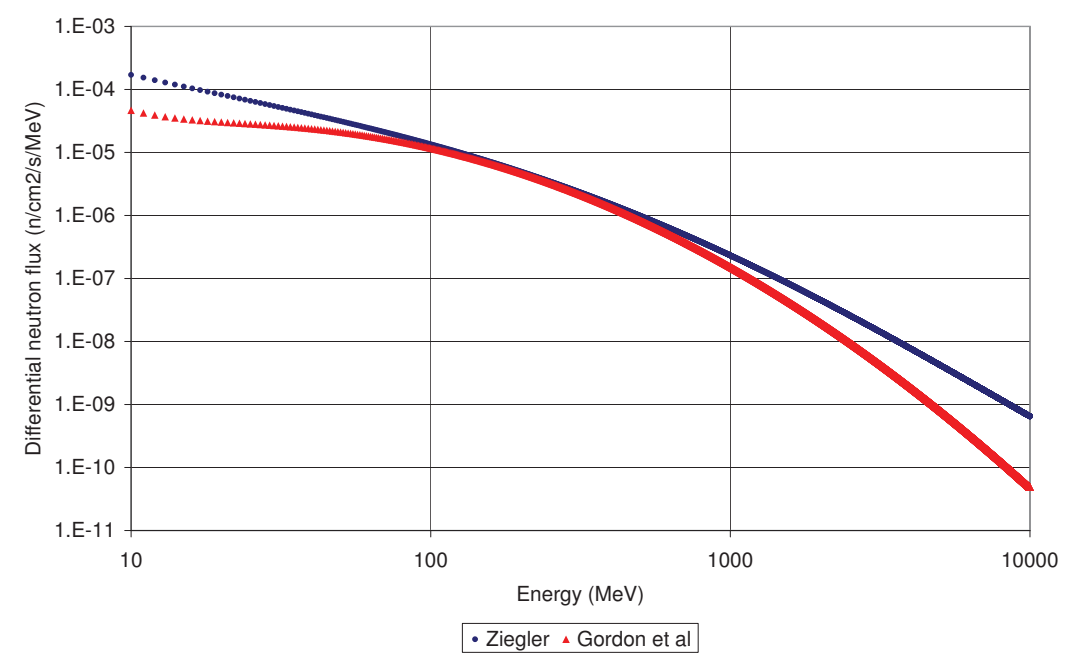

FIGURE 1. Differential flux of cosmic neutrons at sea level, taken from the expressions deduced in the works of Ziegler [16] and Gordon et al. [17].

identify all the reaction products in the final state; the result is in quite good agreement with an upper limit from the IGEX-DM experiment data. Tritium production in other targets was also presented.

- In [22, 23], a 11-g sample of enriched germanium was exposed at Los Alamos Neutron Science Center (LANSCE) to a wide-band pulsed neutron beam that resembles the cosmic-ray neutron flux, with energies up to about 700 $\mathrm{MeV}$. After cooling, germanium gamma counting was performed underground at the Waste Isolation Pilot Plant (WIPP) for $49 \mathrm{~d}$ to evaluate the nuclei production. This production was also predicted by calculating cross sections with CEM03 code. Calculations seem to overestimate production in a factor of 3 depending on isotope (table III in [22]). In addition, measured yields were converted to cosmogenic production rates considering the Gordon et al neutron spectrum (table II in [22]).

- Production cross sections have been measured irradiating at LANSCE a natural germanium target with a proton beam with an energy of $800 \mathrm{MeV}$ [24]. Screening with germanium detectors was performed at Berkeley intermittently from 2 weeks to 5 years after irradiation. A quite good agreement with predictions of Silberberg \& Tsao formulae was obtained.

- Another estimate of production rates after a careful evaluation of excitation functions was presented in [25]. Information on excitation functions for each relevant isotope was collected searching for experimental data (available only for protons) and available calculations (MENDL libraries [12] and other ones based on different codes [13]) and performing some new calculations (using YIELDX). Then, deviation factors were evaluated between measured cross sections and different calculations and the selected description of the excitation functions was the following: HMS-ALICE calculations for neutrons below $150 \mathrm{MeV}$ and YIELDX results above this energy. Production rates were computed for both the Ziegler and Gordon et al spectra (tables 3 and 4 in [25]).

When comparing all the available results for production rates of cosmogenic isotopes in natural and enriched germanium, the found discrepancies can be at least partially understood taking into account the different neutron spectra and the different approaches followed to estimate cross sections. There is an acceptable agreement for ${ }^{60} \mathrm{Co}$ but more scattered results for ${ }^{68} \mathrm{Ge}$.

\section{Tellurium}

Production cross sections of cosmogenic isotopes in tellurium are being studied within the CUORE double beta decay experiment. Results for proton reactions have been recently published [26]. Some measurements were made in USA, by irradiating a tellurium target with the $800 \mathrm{MeV}$ proton beam at LANSCE and performing a gamma screening with germanium detectors in Berkeley. Other measurements were carried out in Europe, exposing $\mathrm{TeO}_{2}$ targets to proton beams with energies of 1.4 and $23 \mathrm{GeV}$ at CERN; first germanium screening was made there for several 
months and later in Milano 2.8 and 4.6 years after irradiation. The obtained cross sections at the three energies are in good agreement with Silberberg \& Tsao predictions (tables 2 and 3 in [26]). In addition, several $\mathrm{TeO}_{2}$ crystals were used as targets for an irradiation at LANSCE with a neutron spectrum that mimics that of the cosmic ray neutrons, from 1.5 to $800 \mathrm{MeV}$. First results for energy-averaged cross sections by neutrons following a germanium screening of $17 \mathrm{~d}$ after irradiation were presented in [27].

\section{Copper and stainless steel}

Copper and stainless steel are materials typically used in rare event experiments. Direct measurements of production rates in both materials were made in Laboratori Nazionali del Gran Sasso (LNGS) [28]. Seven plates made of NOSV grade copper from Nord-deutsche Affinerie AG (Germany), with a total weight of $125 \mathrm{~kg}$, were exposed for $270 \mathrm{~d}$ at an outside hall of the LNGS (altitude $985 \mathrm{~m}$ ) under a roof. Screening with one GeMPI detector was carried out for $103 \mathrm{~d}$. Production rates were derived as the measured saturation activity (table 1 in [28]). The highest values were found for cobalt isotopes; in particular, the measured activity of ${ }^{60} \mathrm{Co}(2100 \pm 190 \mu \mathrm{Bq} / \mathrm{kg})$ greatly exceeded the upper limit derived for the primordial activity. Samples of stainless steel (1.4571 grade) from different batches supplied by Nironit company (with masses from 53 to $61 \mathrm{~kg}$ ) were screened with GeMPI detector at Gran Sasso for the GERDA double beta decay experiment [29]; one of these samples was re-exposed for $314 \mathrm{~d}$ in open air at the LNGS outside laboratory, after a cooling time of $327 \mathrm{~d}$ underground. Production rates were derived for Gran Sasso altitude and scaled down to sea level, considering a correction factor of 2.4 (table 3 in [28]). In this case, ${ }^{60} \mathrm{Co}$ is obscured by anthropogenic contamination, generally present in steel.

Copper is also a specially interesting material because there are many extensive sets of measurements of production cross sections for protons and even for neutrons; this makes it very attractive to compare calculations and experimental data in order to allow a good validation of excitation functions. For this reason, the same study of evaluation of excitation functions and estimate of production rates made for germanium was also carried out for copper [25]. Production rates were calculated using below $100 \mathrm{MeV}$ MENDL2N results for neutrons normalized to the available experimental data when possible, and above that energy experimental data for protons combined with YIELDX calculations when necessary (table 5 in [25]). Results were compared with LNGS measurements, including a correction factor due to altitude and roof estimated to be 2.1 ; very good agreement was found for some isotopes like ${ }^{60} \mathrm{Co}$ but important discrepancies remain for other products.

\section{Other materials}

Cosmogenic activation studies have been performed or are underway for other materials:

- Production rates in sodium iodide are under study in the dark matter ANAIS project. Two new $12.5 \mathrm{~kg} \mathrm{NaI(Tl)}$ detectors produced by Alpha Spectra in USA are taking background data in the Canfranc Underground Laboratory since December 2012, just three days after going underground [30]. Emissions from several cosmogenic isotopes of iodine and tellurium have been identified and their decay observed when comparing the first week data and those taken 75 days later. Preliminary values for the production rates of ${ }^{125} \mathrm{I}_{\left(\mathrm{T}_{1 / 2}=59.4 \mathrm{~d}\right) \text { and }}{ }^{125 m} \mathrm{Te}$ $\left(\mathrm{T}_{1 / 2}=57.4 \mathrm{~d}\right)$ are $615.5 \pm 7.3 \mathrm{~kg}^{-1} \mathrm{~d}^{-1}$ and $82.2 \pm 2.1 \mathrm{~kg}^{-1} \mathrm{~d}^{-1}$ respectively. The theoretical estimate, describing the excitation functions with the MENDL2N library below $100 \mathrm{MeV}$ and the YIELDX code above and considering the Ziegler cosmic neutron spectrum, yields 600 and $79 \mathrm{~kg}^{-1} \mathrm{~d}^{-1}$, in very good agreement with the measurements.

- Production rates have been estimated for natural xenon as made for germanium in [21], with excitation functions calculated using TALYS code and considering the Gordon et al parametrization for the neutron spectrum (table 4 in [21]). Also rates calculated using COSMO and ACTIVIA codes within the XENON100 dark matter experiment were presented in [31]. In addition, individual production cross sections for 271 nuclides have been determined for ${ }^{136} \mathrm{Xe}$ in an inverse kinematics experiment at GSI (tables 1-3 in [32]).

- Even if lead is commonly used in rare event experiments, activation studies on this material are scarce. In [23] first results for some production rates were presented for an irradiation of a lead sample at LANSCE using the neutron beam resembling the cosmic neutron flux and after counting on a low background, underground germanium detector at WIPP. 
- The same experiment performed for tellurium and germanium was also made with a natural molybdenum target, based on $800 \mathrm{MeV}$ proton irradiation at LANSCE and screening with germanium detectors at Berkeley [24].

\section{UNDERGROUND ACTIVATION}

Generation of radioactive isotopes underground is induced mainly by muons. At shallow depths, the capture of negative muons is the relevant process but deep underground interactions by fast muons dominate (direct muon spallation or the electromagnetic and nuclear reactions induced by secondary particles: nucleons, pions, photons,...). Since the muon flux and spectrum depends on depth, it is worth noting that underground activation studies are produced for particular depths; therefore, comparison of different estimates is not straightforward. Some of the main results, most of them related with experiments using large liquid scintillator detectors, are summarized in the following.

- An early estimate of production rates was made in [33] for isotopes induced in materials typically used in neutrino experiments: $\mathrm{C}, \mathrm{O}$ and Ar. Inelastic scattering of muons giving electromagnetic nuclear reactions was considered and rates were evaluated at sea level and underground (2700 m.w.e.) (table I in [33]).

- Production cross sections were measured in a reference experiment [34] performed at CERN irradiating with the SPS muon beam with energies of 100 and $190 \mathrm{GeV}$ different kinds of ${ }^{12} \mathrm{C}$ targets placed behind concrete and water to build the muon shower like in real liquid scintillator experiments. Several detection techniques for measuring products of different half-lives were applied. Then, considering the measured cross-sections (table 5 in [34]) and the deduced dependence with the muon energy $\left(\sigma \propto E_{\mu}^{\alpha}\right.$ with $\left.\alpha=0.73 \pm 0.10\right)$ muon induced background rates for the muon flux at Gran Sasso and BOREXINO detector were computed (table 6 in [34]). Rates for KamLAND were estimated to be a factor $\sim 7$ higher. The most relevant contribution was that of ${ }^{11} \mathrm{C}$.

- In fact, the production rate of ${ }^{11} \mathrm{C}$ was specifically estimated in [35] taking into account all relevant production channels. Evaluation of cross sections from different sources combining data and calculations was made and a FLUKA simulation of monoenergetic muons in Borexino liquid scintillator (trimethilbenzene) was run to derive rates and paths of secondary particles; then, combining this information on the secondaries with the cross sections the individual and total production rates were derived for different muon energies (table II in [35]). Good agreement was found with rates coming from measurements (at 100 and $190 \mathrm{GeV}$ ) [34] and with extrapolations for average muon energies at KamLAND, Borexino and SNOlab.

- Analysis of KamLAND data (from 2002 to 2007) allowed the measurement of production yields [36]. Isotopes were identified and quantified using energy and time information registered. In addition, a FLUKA simulation of monoenergetic muons (in the 10 to $350 \mathrm{GeV}$ range) was performed for KamLAND liquid scintillator to estimate the same yields too (table V in [36]). Comparing with extrapolations (based on the power-law dependance with respect to muon energy) of results from the muon beam experiment [34], some inconsistencies are reported for some isotopes, indicating that estimation by extrapolation might not be sufficient.

- Estimates of production rates for isotopes produced in enriched germanium detectors and set-up materials (cryogenic liquid) were made within the GERDA double beta decay experiment [37], based on a GEANT4 Monte Carlo simulation for the muon spectrum at Gran Sasso. ${ }^{77 m} \mathrm{Ge}$ was identified as the most relevant product.

\section{CONCLUSION}

Cosmogenic activation of materials can jeopardize the sensitivity of ultra-low background experiments due to the production of long-lived isotopes on Earth surface by nucleons and due to the continuous generation of short-lived nuclides deep underground by fast muons. Direct measurements and estimates of production rates and yields for several materials have been made in the context of, for instance, double beta decay and neutrino experiments.

In principle, sea level activation can be kept under control by minimizing exposure at surface and storing materials underground, avoiding flights and even using shields against the hadronic component of cosmic rays. But since these requirements usually complicate the preparation of experiments (for example, crystal growth and mounting of detectors) it would be desirable to have reliable tools to quantify the real danger of exposing the materials to cosmic rays. A good recipe to calculate the production rate of a particular isotope could be the following:

1. To collect all the available information on its production cross section by neutrons and protons, from both measurements (EXFOR database will help) and calculations, either from libraries (HEAP-2009 is a recent, wide- 
range compilation) or using codes (based on semiempirical formulae like YIELDX and ACTIVIA or on Monte Carlo simulation like CEM).

2. To choose the best description of the excitation functions of products over the whole energy range, by calculating deviation factors between measurements and calculations.

3. To calculate the production rates considering the preferred cosmic ray spectrum and to compare them with previous estimates or measurements if available.

Uncertainties in this kind of calculations (hardly lower than 50\%) come mainly from the difficulties encountered on the accurate description of cosmic ray spectra and on the precise evaluation of inclusive production cross-sections in all the relevant energy range; the low and medium energy regions below a few hundreds of $\mathrm{MeV}$ are the most problematic ones since neutron data are scarce and differences between neutron and proton cross sections may be important.

\section{REFERENCES}

1. G. Heusser, Annu. Rev. Nucl. Part. Sci. 45, 543 (1995).

2. J. A. Formaggio and C. J. Martoff, Annu. Rev. Nucl. Part. Sci. 54, 361 (2004).

3. http://www.nndc.bnl.gov/exfor/exfor00.htm, http://www-nds.iaea.org/exfor/exfor.htm

4. R. Silberberg and C. H. Tsao, Astrophys. J. Suppl. Ser. 25, 315 (1973); ibid p. 335.

5. R. Silberberg and C. H. Tsao, Astrophys. J. Suppl. Ser. 35, 129 (1977); Astrophys. J. Suppl. Ser. 58, 873 (1985); Phys. Rep. 191, 351 (1990).

6. R. Silberberg and C. H. Tsao, Astrophys. J. 501, 911 (1998).

7. C. J. Martoff and P. D. Lewin, Comput. Phys. Commun. 72, 96 (1992).

8. J. J. Back and Y. A. Ramachers, Nucl. Instrum. Meth. A 586, 286 (2008).

9. Yu. A. Korovin et al., Nucl. Instrum. Meth. A 624, 20-26 (2010).

10. Yu. A. Titarenko et al., Phys. Rev. C 84, 064612 (2011).

11. http://www-nds.iaea.org/public/rnal/www/

12. http://www-nds.iaea.org/publications/iaea-nds/iaea-nds-0136.htm, http://www.oecd-nea.org/tools/abstract/detail/iaea1376

13. K. A. Van Riper, S. G. Mashnik and W. B. Wilson, Nucl. Instrum. Meth. A 463, 576-585 (2001).

14. W. N. Hess, H. W. Patterson and R. Wallace, Phys. Rev. 116, 449 (1959).

15. D. Lal and B. Peters, Cosmic ray produced radioactivity on the Earth, Springer, Berlin-Heidelberg, 1967.

16. J. F. Ziegler, IBM J. Res. Develop. 42, 117-140 (1998).

17. M. S. Gordon et al., IEEE Transactions on Nuclear Science 513427 (2004).

18. F. T. Avignone et al., Nucl. Phys. B (Proc. Suppl.) 28A, 280 (1992).

19. H. Miley et al., Nucl. Phys. B (Proc. Suppl.) 28A, 212 (1992).

20. I. Barabanov et al., Nucl. Instrum. Meth. B 251, 115-120 (2006).

21. D. M. Mei et al., Astropart. Phys. 31, 417-420 (2009).

22. S. R. Elliott et al., Phys. Rev. C 82, 054610 (2010).

23. V. E. Guiseppe, CAB Workshop (Berkeley 2011), https://docs.sanfordlab.org/docushare/dsweb/View/Wiki141/Agenda/guiseppe_berkeley_apr11_V2.pdf

24. E. B. Norman et al., Nucl. Phys. B (Proc. Suppl.) 143, 508 (2005). E. B. Norman et al., CAB Workshop (Berkeley 2011) https://docs.sanfordlab.org/docushare/dsweb/View/Wiki141/Agenda/Radionuclide $\% 20$ Production $\% 20$ from $\% 20 \% 20$ Proton $\% 20$ Interactions $\% 20 \mathrm{in} \% 20 \mathrm{Ge}, \mathrm{Mo}$, Te.pdf

25. S. Cebrián et al., Astropart. Phys. 33 316-329 (2010).

26. A. F. Barghouty et al., Nucl. Instrum. Meth. B 295, 16 Ü21 (2013).

27. B. Wang, CAB workshop (Berkeley 2011), https://docs.sanfordlab.org/docushare/dsweb/View/Wiki-141/Agenda/CrosssectionMeasurementsTeO2_4_13_2011.pdf

28. M. Labustenstein, G. Heusser, App. Rad. Isot. 67, 750-754 (2009).

29. W. Maneschg et al., Nucl. Instrum. Meth. A 593, 448 Ü-453 (2008).

30. J. Amaré et al., these proceedings. C. Cuesta, "ANAIS-0: Feasibility study for a $250 \mathrm{~kg} \mathrm{NaI(Tl)} \mathrm{dark} \mathrm{matter} \mathrm{search} \mathrm{experiment}$ at the Canfranc Underground Laboratory", PhD thesis, May 2013.

31. A. Kish, CAB workshop (Berkeley 2011), https://docs.sanfordlab.org/docushare/dsweb/View/Wiki141/Agenda/Berkeley2011_Xe100_Kish_red4.pdf

32. L. Giot et al., Nucl. Phys. A 899, 116-Ü132 (2013).

33. J. S. O'Connell and F. J. Schima, Phys. Rev. D 38, 2277-2279 (1988).

34. T. Hagner et al., Astropart. Phys. 14, 33-47 (2000).

35. C. Galbiatti et al., Phys. Rev. C 71, 055805 (2005).

36. S. Abe et al., Phys. Rev. C 81, 025807 (2010).

37. L. Pandola et al., Nucl. Instrum. Meth. A 570, 149-158 (2007). 
Copyright of AIP Conference Proceedings is the property of American Institute of Physics and its content may not be copied or emailed to multiple sites or posted to a listserv without the copyright holder's express written permission. However, users may print, download, or email articles for individual use. 